\title{
A NATURAL FAMILY OF FLAG MATROIDS
}

\author{
ANNA DE MIER
}

\begin{abstract}
A flag matroid can be viewed as a chain of matroids linked by quotients. Flag matroids, of which relatively few interesting families have previously been known, are a particular class of Coxeter matroids. In this paper we give a family of flag matroids arising from an enumeration problem that is a generalization of the tennis ball problem. These flag matroids can also be defined in terms of lattice paths and they provide a generalization of the lattice path matroids of [Bonin et al., JCTA 104 (2003)].
\end{abstract}

\section{INTRODUCTION AND PRELIMINARIES}

Flag matroids are a subclass of Coxeter matroids, but they can also be described in pure matroid-theoretical terms. Roughly speaking, a flag matroid is a collection of matroids on the same ground set that form a chain in the strong order (i.e., they are quotients of each other). Flag matroids play an important role in the theory of Coxeter matroids and also shed light on ordinary matroid theory. Nevertheless, not many classes of flag matroids have been studied up to now. The goal of this paper is to introduce a new family of flag matroids based on an enumeration problem and show how these flag matroids can be interpreted in terms of lattice paths. We refer to [4, especially to Chapter 1, for an introduction to flag matroids and the ideas behind them.

We assume the reader is familiar with the basic concepts of matroid theory; we follow the notation of Oxley's book [8]. We recall here only the notion of quotient. Given two matroids $M$ and $N$ on the same ground set, $M$ is a quotient of $N$ if every flat of $M$ is a flat of $N$ (one can also say that $M$ is a strong map image of $N)$. In this case, the rank of $M$ is at most the rank of $N$, with equality holding if and only if $M$ and $N$ are equal.

We also need to say a few words about lattice path matroids. We do not need lattice path matroids in general as defined in [2, but only the subclass of nested matroids. These matroids have independently arisen several times in the literature since at least 1965, and have been given a variety of names; see [1, 2, and the references therein for definitions and results (in these papers, nested matroids are called "generalized Catalan matroids").

Let $P$ be a lattice path from $(0,0)$ to $(m, r)$ with steps $E=(1,0)$ and $N=(0,1)$. Let $\mathcal{P}$ be the set of paths from $(0,0)$ to $(m, r)$ with steps $E$ and $N$ and that do not

Date: September 16, 2018.

1991 Mathematics Subject Classification. Primary: 05B35.

Key words and phrases. matroid, flag matroid, lattice path matroid, tennis ball problem. 
go above $P$. For each path $Q \in \mathcal{P}$, let $Q_{N}=\{i$ : step $i$ in $Q$ is $N\}$. We denote by $[n]$ the set $\{1,2, \ldots, n\}$.

Theorem 1.1. The set $\left\{Q_{N}: Q \in \mathcal{P}\right\}$ is the collection of bases of a matroid $M[P]$ on the ground set $[m+r]$.

A matroid is nested if it is isomorphic to $M[P]$ for some path $P$. Hence, the bases of a nested matroid are in bijection with the lattice paths that do not go above a certain fixed path $P$ (see the left side of Figure 1 for an example of a nested matroid on the set [15]; the path highlighted corresponds to the basis $\{2,5,8,11,12,15\})$. The name nested comes from the fact that a nested matroid can also be defined as a transversal matroid whose presentation consists of nested sets, and also because of the following characterization of nested matroids in terms of cyclic flats (recall that a flat is cyclic if it is a union of circuits).

Theorem 1.2. A matroid is nested if and only if its cyclic flats form a chain under inclusion. Furthermore, the proper non-trivial cyclic flats of the matroid $M[P]$ are the initial segments $[t]$ of $[m+r]$, where $t$ is such that step $t$ of $P$ is $E$ and step $t+1$ is $N$.

Our view on flag matroids is slightly different from that of [4], but it is easy to see that the two perspectives are equivalent. The definition in [4] is in terms of flags of sets, whereas ours relies on what we call ordered partitions of a set. For the reader already familiar with the theory of flag matroids, changing from one definition to the other should be straightforward.

Definition 1.3. An ordered $k$-partition of a set $S$ is a $k$-tuple $\left(A_{1}, \ldots, A_{k}\right)$ of non-empty sets with $A_{1} \cup A_{2} \cup \cdots \cup A_{k}=S$ and $A_{i} \cap A_{j}=\emptyset$ whenever $i \neq j$. For positive integers $r_{1}, \ldots, r_{k}$ such that $r_{1}+\cdots+r_{k}=|S|$, an $\left(r_{1}, r_{2}, \ldots, r_{k}\right)$-partition of $S$ is an ordered $k$-partition $\left(A_{1}, \ldots, A_{k}\right)$ of $S$ such that $\left|A_{i}\right|=r_{i}$ for all $i$ with $1 \leq i \leq k$.

The bases of a matroid $M$ on a set $S$ trivially determine a collection of ordered 2-partitions of $S$ : take all pairs of the form $(B, S-B)$, where $B$ is a basis of $M$. The first axiom for flag matroids generalizes this idea; the other two axioms arise from the definition of a flag matroid in terms of Coxeter groups (see 44). Given an ordered $k$-partition $B$, we denote by $B_{i}$ the $i$-th set in the $k$-tuple $B$.

Definition 1.4. A flag matroid $F$ is pair $(S, \mathcal{F})$ such that $\mathcal{F}$ is a collection of ordered $k$-partitions of the set $S$ satisfying the following properties:

(F1) for $1 \leq i \leq k$, the set $\mathcal{B}_{i}=\left\{\cup_{1 \leq j \leq i} B_{j}: B \in \mathcal{F}\right\}$ is the set of bases of a matroid $M_{i}$

(F2) for $1 \leq i \leq k-1, M_{i}$ is a quotient of $M_{i+1}$;

(F3) if $\left(A_{1}, \ldots, A_{k}\right)$ is an ordered $k$-partition of $S$ such that, for all $i$ with $1 \leq$ $i \leq k$, the set $A_{1} \cup \cdots \cup A_{i}$ is a basis of the matroid $M_{i}$, then $\left(A_{1}, \ldots, A_{k}\right)$ is in $\mathcal{F}$.

Because of the similarity with matroids, we call the elements of $\mathcal{F}$ the flag bases of $F$. Note that it follows from the definition that there exist integers $r_{1}, \ldots, r_{k}$ 
adding up to $|S|$ such that all ordered partitions in $\mathcal{F}$ are in fact $\left(r_{1}, \ldots, r_{k}\right)$ partitions. The $k$-tuple $\left(r_{1}, \ldots, r_{k}\right)$ will be called the flag rank of $F$. The matroids $M_{1}, \ldots M_{k}$ above are called the constitutents of the flag matroid $F$. Notice that $M_{k}$ is the free matroid on $S$ and that $M_{i}$ has rank $r_{1}+\cdots+r_{i}$.

A trivial example of a flag matroid is the uniform flag matroid, having as flag bases all possible $\left(r_{1}, \ldots, r_{k}\right)$-partitions of a set $S$. Other examples come from chains of subspaces of a vector space, giving rise to representable flag matroids. Also, given a matroid $M$, the underlying flag matroid has as constituents the matroids $M_{i}=T^{i}(M)$, the truncations of $M$ to ranks 1 to $r(M)$. A flag matroid with flag rank $(1,1, \ldots, 1)$ can also be viewed as a Gaussian greedoid [5].

Flag matroids, as is true of Coxeter matroids in general, are usually viewed in terms of their polytopes. For instance, the polytope of the uniform flag matroid of flag rank $(1,1, \ldots, 1)$ is the permutahedron; the polytope of the underlying flag matroid is studied in [3].

\section{The Tennis Ball Problem}

The tennis ball problem is a problem in enumeration that can be phrased in terms of balls-and-bins and in terms of lattice paths. We need both approaches here. We first define the original problem and show its solution amounts to counting bases of a certain type of nested matroid. Then we generalize the problem and show that it gives rise to a family of flag matroids.

Definition 2.1. Let $l_{1}$ and $l_{2}$ be positive integers. Suppose we have infinitely many balls numbered $1,2, \ldots$ and two bins labelled $A$ and $B$. In the first turn, balls $1,2, \ldots, l_{1}+l_{2}$ go into bin $A$, and then $l_{2}$ of those are moved to bin $B$. In the second turn, balls $l_{1}+l_{2}+1, \ldots, 2\left(l_{1}+l_{2}\right)$ go into bin $A$, and of the $2 l_{1}+l_{2}$ balls there, $l_{2}$ are moved to bin $B$. At each turn, the next $l_{1}+l_{2}$ balls go into bin $A$, and of the balls in $A, l_{2}$ are moved to bin $B$. An $n$-configuration is an ordered 2-partition of $\left[\left(l_{1}+l_{2}\right) n\right]$ giving a possible distribution of balls in the bins after $n$ turns. The $\left(l_{1}, l_{2}\right)$-tennis ball problem asks for the number of $n$-configurations.

This problem was solved in [7] using the following relationship with nested matroids.

Theorem 2.2. The number of $n$-configurations of the $\left(l_{1}, l_{2}\right)$-tennis ball problem is the number of bases of the nested matroid $M\left[\left(N^{l_{1}} E^{l_{2}}\right)^{n}\right]$.

The proof is straightforward by the bijection that sends a basis $\left\{n_{1}, \ldots, n_{r}\right\}$ of $M\left[\left(N^{l_{1}} E^{l_{2}}\right)^{n}\right]$ to the configuration having the balls $\left\{n_{1}, \ldots, n_{r}\right\}$ in bin $A$ (see Figure 1). For non-negative integers $a, b$, the matroid $M\left[\left(N^{a} E^{b}\right)^{n}\right]$ is called in the sequel the $n$-th $(a, b)$-tbp matroid.

Theorem 2.2 can be rephrased by saying that the tennis ball problem with two bins gives the bases of a matroid. The main result of this section is that the tennis ball problem with $k$ bins, that we next define, gives the flag bases of a flag matroid.

Definition 2.3. Let $\left(l_{1}, l_{2}, \ldots, l_{k}\right)$ be a $k$-tuple of positive integers; let $L=l_{1}+$ $l_{2}+\cdots+l_{k}$. Suppose we have infinitely many balls numbered $1,2, \ldots$ and $k$ bins labelled $\Gamma_{1}, \Gamma_{2}, \ldots, \Gamma_{k}$. In the first turn, balls $1,2, \ldots, L$ go into bin $\Gamma_{1}$; of those, 


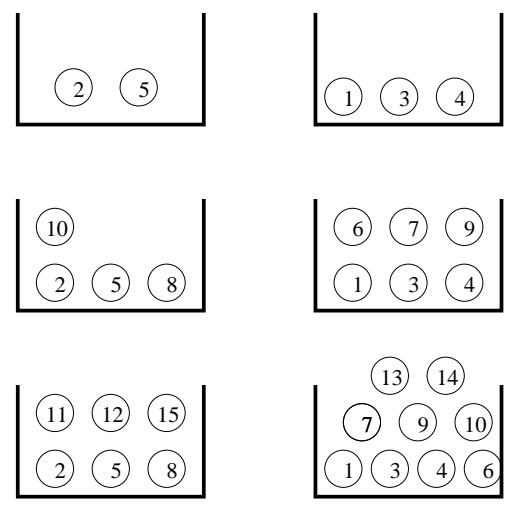

Figure 1. A diagram representing the matroid $M\left[\left(N^{2} E^{3}\right)^{3}\right]$. The path highlighted corresponds to the 3-configuration shown on the right.

$L-l_{1}$ are moved to bin $\Gamma_{2}$; of those, $L-l_{1}-l_{2}$ are moved to bin $\Gamma_{3}$, and so on until $l_{k}$ balls are moved to bin $\Gamma_{k}$. In the second turn, balls $L+1, \ldots, 2 L$ go into bin $\Gamma_{1}$, and of the $l_{1}+L$ balls there, $L-l_{1}$ are moved to bin $\Gamma_{2}$; of the balls now in bin $\Gamma_{2}$, $L-l_{1}-l_{2}$ are moved to bin $\Gamma_{3}$, and so on. At each turn, the next $L$ balls go into bin $\Gamma_{1}$, and of the balls in $\Gamma_{1}, L-l_{1}$ are moved to bin $\Gamma_{2}$, etc... An $n$-configuration is an ordered $k$-partition of $[L n]$ corresponding to a possible distribution of the balls in the bins after $n$ turns. The $\left(l_{1}, \ldots, l_{k}\right)$-tennis ball problem asks for the number of $n$-configurations, that is, the number of $\left(n l_{1}, n l_{2}, \ldots, n l_{k}\right)$-partitions of the set $[n L]$ that we can obtain after $n$ turns.

Note that we are only interested in $n$-configurations, not in the movements of the balls that lead to them; an $n$-configuration can typically be obtained by several different movements of the balls. Let $F_{n}^{\left(l_{1}, \ldots, l_{k}\right)}$ be the collection of $\left(n l_{1}, \ldots, n l_{k}\right)$ partitions of $[n L]$ that we get as $n$-configurations. These ordered partitions are the flag bases of a flag matroid whose constituent matroids are nested matroids.

Theorem 2.4. The set $F_{n}^{\left(l_{1}, \ldots, l_{k}\right)}$ is the collection of flag bases of a flag matroid on the set $[n L]$. Moreover, for $i$ with $1 \leq i \leq k$, the $i$-th constituent of the flag matroid is the $n$-th $\left(l_{1}+\cdots+l_{i}, l_{i+1}+\cdots+l_{k}\right)$-tbp matroid.

Proof. We need to check that axioms (F1)-(F3) hold for $\mathcal{F}=F_{n}^{\left(l_{1}, \ldots, l_{k}\right)}$. It is easy to see that the set $\mathcal{B}_{i}=\left\{\cup_{1 \leq j \leq i} B_{j}: B \in \mathcal{F}\right\}$ is the set of bases of the $n$-th $\left(l_{1}+\cdots+l_{i}, l_{i+1}+\cdots+l_{k}\right)$-tbp matroid, so (F1) holds.

To show that (F2) holds it is enough to prove that if $a+b=a^{\prime}+b^{\prime}$ and $a<a^{\prime}$, then the $n$-th $(a, b)$-tbp matroid $M$ is a quotient of the $n$-th $\left(a^{\prime}, b^{\prime}\right)$-tbp matroid $M^{\prime}$. We show that each flat $F$ of $M$ is a flat of $M^{\prime}$. If $F$ is a cyclic flat, this follows from the characterization of cyclic flats of nested matroids in Theorem 1.2. Otherwise, $F$ is $F^{\prime} \cup I$, where $F^{\prime}$ is a cyclic flat of $M$ and $I$ is the set of isthmuses of $F$. So $F^{\prime}$ is an initial segment of $[(a+b) n]$ whose length is a multiple of $a+b$. Since $a+b=a^{\prime}+b^{\prime}$, by Theorem 1.2 again we have that $F^{\prime}$ is a cyclic flat of $M^{\prime}$. For $F^{\prime} \cup I$ to be a flat of $M$, the set $I$ has to be such that $|I \cap[t(a+b)]|<a$ for 
all $t$. Since $a+b=a^{\prime}+b^{\prime}$ and $a<a^{\prime}$, we also have that $\left|I \cap\left[t\left(a^{\prime}+b^{\prime}\right)\right]\right|<a^{\prime}$ for all $t$, hence $F^{\prime} \cup I$ is a flat of $M^{\prime}$.

To show that (F3) holds, let $\left(A_{1}, \ldots, A_{k}\right)$ be an $\left(n l_{1}, \ldots, n l_{k}\right)$-partition of $[n L]$ such that for all $i, \cup_{1 \leq j \leq i} A_{j}$ is a basis of the $n$-th $\left(l_{1}+\cdots+l_{i}, l_{i+1}+\cdots+l_{k}\right)$-tbp matroid. We show that $\left(A_{1}, \ldots, A_{k}\right)$ is in the collection $\mathcal{F}$ by showing how to get the $n$-configuration $\left(A_{1}, \ldots, A_{k}\right)$ by suitably moving the balls. Let $C_{i}$ be $\cup_{j=1}^{i} A_{j}$. To avoid wordiness, we identify balls with the integers of their labels. We start with all bins empty and explain how to perform $n$ turns with the condition that at the end of each turn, the set of balls in bin $\Gamma_{i}$ is a subset of $[n L]-C_{i-1}$, for all $i$ with $1 \leq i \leq k$. Suppose we have performed $t-1$ such turns, for $t$ with $0<t \leq n$, and let us describe turn $t$. The assumption that $C_{k-1}, C_{k-2}, \ldots, C_{1}$ are bases of their respective tbp matroids gives the following facts:

$(\mathbf{k}-\mathbf{1})$ At least $t l_{k}$ of the elements of $[t L]$ are in $[t L]-C_{k-1}$;

$(\mathbf{k}-\mathbf{2})$ at least $t\left(l_{k}+l_{k-1}\right)$ of the elements of $[t L]$ are in $[t L]-C_{k-2}$;

(2) at least $t\left(l_{k}+\cdots+l_{3}\right)$ of the elements of $[t L]$ are in $[t L]-C_{2}$;

(1) at least $t\left(l_{k}+\cdots+l_{2}\right)$ of the elements of $[t L]$ are in $[t L]-C_{1}$.

Since after the first $t-1$ turns there are $(t-1) l_{j}$ elements in bin $\Gamma_{j}$, we can deduce from facts $(1)-(k-1)$ that at this point, for all $i$ with $1 \leq i \leq k-1$, at least $l_{i+1}+\cdots+l_{k}$ integers from $[t L]$ are in $[t L]-C_{i}$ but not in $\Gamma_{i+1} \cup \cdots \cup \Gamma_{k}$.

Now move $(t-1) n+1,(t-1) n+2, \ldots, t n$ to bin $\Gamma_{1}$. Of all the integers in $\Gamma_{1}$, choose $L-l_{1}$ to move to bin $\Gamma_{2}$ starting by as many as possible of the ones in $[t L]-C_{k-1}$ that are still in $\Gamma_{1}$, then take as many as possible of the ones in $[t L]-C_{k-2}$, and so on until having $L-l_{1}$ integers. The remarks in the previous paragraph show that it is possible to choose integers in this way. We move them to bin $\Gamma_{2}$. Since $[t L]-C_{k-1} \subset[t L]-C_{k-2} \subset \cdots \subset[t L]-C_{1}$, the integers now in $\Gamma_{2}$ are a subset of $[t L]-C_{1}$, as required. Moreover, by the way the balls are chosen, we have that among the balls that are at this point in $\Gamma_{2}$, at least $l_{3}+\cdots+l_{k}$ are in $[t L]-C_{2}$.

We describe generally how to move $l_{i}+\cdots+l_{k}$ balls to bin $\Gamma_{i}$ from bin $\Gamma_{i-1}$ in a way such that the balls in $\Gamma_{i}$ are a subset of $[t L]-C_{i-1}$, and, moreover, at least $l_{i+1}+\cdots+l_{k}$ of them are in $[t L]-C_{i}$. From the balls in bin $\Gamma_{i-1}$, pick $l_{i}+\cdots+l_{k}$ starting by as many as possible from $[t L]-C_{k-1}$; if there are not still $l_{i}+\cdots+l_{k}$, then take as many as possible from $[t L]-C_{k-2}$, and so on, until taking as many as possible from $[t L]-C_{i-1}$. By the same reason as above, such integers exist; the integers now in $\Gamma_{i}$ are a subset of $[t L]-C_{i-1}$ and at least $l_{i+1}+\cdots+l_{k}$ of them are in $[t L]-C_{i}$.

At the end of $n$ turns, we have $n l_{i}$ balls in bin $\Gamma_{i}$, and these are a subset of $[n L]-C_{i-1}$, for all $i$. This implies that bin $\Gamma_{k}$ contains exactly the balls in $A_{k}$, and hence bin $\Gamma_{k-1}$ contains the balls in $A_{k-1}$, and so on. Therefore the ordered partition $\left(A_{1}, \ldots, A_{k}\right)$ is an $n$-configuration, thus it belongs to the collection $F_{n}^{\left(l_{1}, \ldots, l_{k}\right)}$ and (F3) follows. 


\section{INTERPRETATION IN TERMS OF LATTICE PATHS}

The tennis ball problem with two bins has a simple interpretation in terms of lattice paths; we associate bin $A$ with steps $N$ and bin $B$ with steps $E$, and then each $n$-configuration corresponds to a path that does not go above $\left(N^{l_{1}} E^{l_{2}}\right)^{n}$. For the tennis ball problem with $k$ bins, we can associate to each bin a direction in $\mathbb{N}^{k}$. Then the flag bases of $F_{n}^{\left(l_{1}, \ldots, l_{k}\right)}$ are in bijection with certain paths in $\mathbb{N}^{k}$. We characterize those paths combinatorially and for $k=3$ we describe them as the set of lattice paths that do not cross a certain border.

Let $e_{1}, \ldots, e_{k}$ be the unit coordinate vectors in $\mathbb{R}^{k}$. To each $n$-configuration of the $\left(l_{1}, \ldots, l_{k}\right)$-tennis ball problem, we associate a path $s_{1} s_{2} \cdots s_{n L}$ from $(0, \ldots, 0)$ to $\left(n l_{1}, \ldots, n l_{k}\right)$ with steps defined as $s_{i}=e_{j}$ if ball $i$ is in bin $\Gamma_{j}$. We call this path an $n$-configuration path. Hence, an $n$-configuration path can be seen as a sequence of elements from $\left\{e_{1}, \ldots, e_{k}\right\}$. It is easy to characterize which such sequences give configuration paths.

Lemma 3.1. A path from $(0, \ldots, 0)$ to $\left(n l_{1}, \ldots, n l_{k}\right)$ is an $n$-configuration path for the $\left(l_{1}, \ldots, l_{k}\right)$-tennis ball problem if and only if, for all $t$ with $1 \leq t \leq n$ and all $i$ with $1 \leq i \leq k-1$, among the first $t L$ steps there are at most $t\left(l_{1}+\cdots+l_{i}\right)$ whose type belongs to $\left\{e_{1}, \ldots, e_{i}\right\}$.

Proof. After $t$ turns, for $1 \leq t \leq n$, there are exactly $t\left(l_{1}+\cdots+l_{i}\right)$ balls of $[t L]$ in the first $i$ bins. Since balls can only move to bins with a higher index, at the end of $n$ turns there are at most $t\left(l_{1}+\cdots+l_{i}\right)$ balls of $[t L]$ in $\Gamma_{1} \cup \cdots \cup \Gamma_{i}$. Hence among the first $t L$ steps of a configuration path there are at most $t\left(l_{1}+\cdots+l_{i}\right)$ steps whose type is in $\left\{e_{1}, \ldots, e_{i}\right\}$.

For the converse, assume we have a path $\pi$ that satisfies the condition. Let $A_{i}$ be the set of integers $s$ such that step $s$ in $\pi$ is of type $e_{i}$. Consider the $\left(n l_{1}, \ldots, n l_{k}\right)$-partition $\left(A_{1}, \ldots, A_{k}\right)$ of $[n L]$ obtained in this way. The condition on the path implies that the set $\cup_{j=1}^{i} A_{j}$ is a basis of the $\left(l_{1}+\ldots+l_{i}, l_{i+1}+\ldots+l_{k}\right)$ tbp matroid, for all $i$ with $1 \leq i \leq k-1$. Therefore $\left(A_{1}, \ldots, A_{k}\right)$ is a flag basis of the flag matroid $F_{n}^{\left(l_{1}, \ldots, l_{k}\right)}$ and hence $\pi$ is an $n$-configuration path, as required.

The following is an immediate corollary.

Corollary 3.2. Given an n-configuration path $\pi$, the path obtained by switching a pair of steps $s_{i}=e_{l}$ and $s_{j}=e_{m}$ is a configuration path if $i<j$ and $l \leq m$. Moreover, let $\pi^{\prime}$ be an initial segment of $\pi$ with $t_{j}^{\prime}$ steps of type $e_{j}$ for all $1 \leq j \leq k$. Let $n^{\prime}$ be the minimum integer such that $t_{j}^{\prime} \leq n^{\prime} l_{j}$ for all $j$. For $n^{\prime \prime} \geq 0$, consider the path obtained from $\pi^{\prime}$ followed by $\left(n^{\prime}+n^{\prime \prime}\right) l_{k}-t_{k}^{\prime}$ steps $e_{k}$, then $\left(n^{\prime}+n^{\prime \prime}\right) l_{k-1}-t_{k-1}^{\prime}$ steps $e_{k-1}$, and so on, until finishing with $\left(n^{\prime}+n^{\prime \prime}\right) l_{1}-t_{1}^{\prime}$ steps $e_{1}$. Then this path is an $\left(n^{\prime}+n^{\prime \prime}\right)$-configuration path.

The $n$-diagram for the $\left(l_{1}, \ldots, l_{k}\right)$-tennis ball problem is the set of points in $\mathbb{N}^{k}$ that are contained in some $n$-configuration path. Our goal is to study what these diagrams look like for $k=3$. The following is another corollary of Lemma 3.1 .

Corollary 3.3. If $(x, y, z)$ is in the $n$-diagram, then $\left(x^{\prime}, y, z^{\prime}\right)$ is in the $n$-diagram for all $z \leq z^{\prime} \leq n l_{3}$ and all $x^{\prime} \leq x$. 
A direct consequence of this corollary is that to describe the $n$-diagram it is enough to give, for each $(x, y)$, the minimum value of $z$ such that $(x, y, z)$ is a point of the $n$-diagram; this minimum is denoted $m_{n}(x, y)$. (Trivially the maximum value of $z$ is $n l_{3}$.) If no such $z$ exists, because no point of the form $(x, y, *)$ is in the $n$-diagram, we set $m_{n}(x, y)=*$. So the $n$-diagram is described by an $\left(n l_{1}+1\right) \times\left(n l_{2}+1\right)$ matrix $\mathcal{M}_{n}$ with entries in the set $\left\{0,1, \ldots, n l_{3}\right\} \cup\{*\}$ and such that in row $x$ and column $y$ we have $m_{n}(x-1, y-1)$. If $n=1$, then trivially $\mathcal{M}_{1}$ is the zero matrix and the corresponding 1-diagram is represented in Figure 2 . In all figures below, the direction of the third coordinate has been reversed for a better view of the picture, and, as pointed out above, all points under a point that is shown are in the diagram as well.

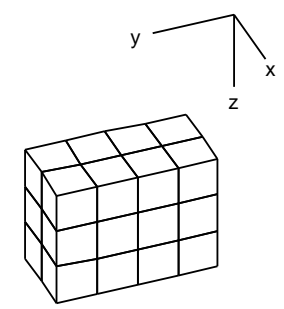

Figure 2. The 1-diagram for the $(2,4,3)$-tennis ball problem.

The 2-diagram for the (2,4,3)-tennis ball problem is shown in Figure 3. We first give the matrices $\mathcal{M}_{n}$ and then prove that they give the right diagrams.

The matrix $\mathcal{M}_{2}$ is made up of four blocks,

$$
\mathcal{M}_{2}=\left(\begin{array}{l|l}
A & B \\
\hline C & D
\end{array}\right)
$$

where $A$ is the $\left(l_{1}+1\right) \times\left(l_{2}+1\right)$ matrix all whose entries are zero (hence, it is $\left.\mathcal{M}_{1}\right), D$ is an $l_{1} \times l_{2}$ matrix all whose entries are $l_{3}, B$ is an $\left(l_{1}+1\right) \times l_{2}$ matrix with

$$
b_{i, j}= \begin{cases}0 & \text { if } i \leq l_{1}+1-j, \\ l_{3} & \text { otherwise }\end{cases}
$$

and $C$ is the $l_{1} \times\left(l_{2}+1\right)$ matrix with $\min \left\{l_{2}, l_{3}\right\}+1$ non-* columns, with the elements in the last column being $l_{3}$ and each other non-* column being obtained by adding +1 to the next, that is,

$$
\left(\begin{array}{ccccccccc}
* & \cdots & * & 2 l_{3} & 2 l_{3}-1 & 2 l_{3}-2 & \cdots & l_{3}+1 & l_{3} \\
\vdots & \ddots & \vdots & \vdots & \vdots & \vdots & \ddots & \vdots & \vdots \\
* & \cdots & * & 2 l_{3} & 2 l_{3}-1 & 2 l_{3}-2 & \cdots & l_{3}+1 & l_{3}
\end{array}\right)
$$



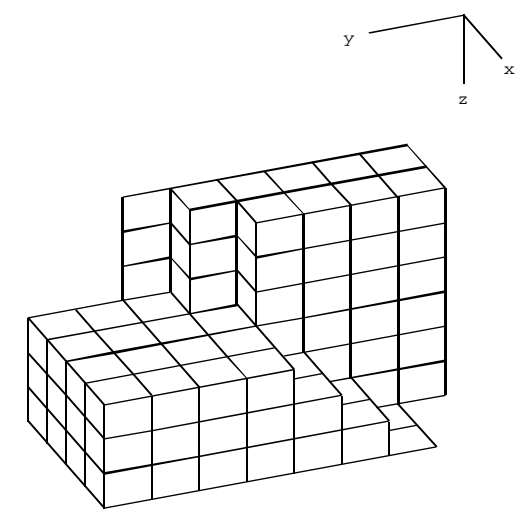

Figure 3. The 2-diagram for the (2,4,3)-tennis ball problem.

We now define recursively the $\left(n l_{1}+1\right) \times\left(n l_{2}+1\right)$ matrix $\mathcal{M}_{n}$ that gives the $n$-diagram. The matrix $\mathcal{M}_{n}$ also decomposes into 4 blocks

$$
\mathcal{M}_{n}=\left(\begin{array}{c|c}
A_{n} & B_{n} \\
\hline C_{n} & D_{n}
\end{array}\right)
$$

where $A_{n}$ has $(n-1) l_{1}+1$ rows and $(n-1) l_{2}+1$ columns. The matrix $A_{n}$ is $\mathcal{M}_{n-1}$. The entry in row $i$ and column $j$ of the matrix $B_{n}$, for $1 \leq i \leq(n-1) l_{1}+1$ and $1 \leq j \leq l_{2}$, is given by $(n-s) l_{3}$, where $s$ is the only integer for which $(n-s)\left(l_{1}+l_{2}\right)<i-1+j+(n-1) l_{2} \leq(n-s+1)\left(l_{1}+l_{2}\right)$. Roughly speaking, $B_{n}$ consists of diagonal stripes of width $l_{1}+l_{2}$, see the examples below. The matrix $C_{n}$ has $l_{1}$ rows and $(n-1) l_{2}+1$ columns; all entries in the last column are $(n-1) l_{3}$ and each column is obtained by adding one to the next, until we reach $n l_{3}$; hence it is given by

$$
\left(\begin{array}{ccccccccc}
* & \cdots & * & n l_{3} & n l_{3}-1 & n l_{3}-2 & \cdots & (n-1) l_{3}+1 & (n-1) l_{3} \\
\vdots & \ddots & \vdots & \vdots & \vdots & \vdots & \ddots & \vdots & \vdots \\
* & \cdots & * & n l_{3} & n l_{3}-1 & n l_{3}-2 & \cdots & (n-1) l_{3}+1 & (n-1) l_{3}
\end{array}\right)
$$

Finally, $D_{n}$ is the $l_{1} \times l_{2}$ matrix all whose entries are $(n-1) l_{3}$. The matrix $\mathcal{M}_{3}$ is shown below for the $(2,4,3)$ - and the $(3,2,2)$-tennis ball problems, and the corresponding 3-diagrams are shown in Figures 4 and 5. 


$$
\left(\begin{array}{ccccc|cccc|cccc}
0 & 0 & 0 & 0 & 0 & 0 & 0 & 3 & 3 & 3 & 3 & 3 & 3 \\
0 & 0 & 0 & 0 & 0 & 0 & 3 & 3 & 3 & 3 & 3 & 3 & 6 \\
0 & 0 & 0 & 0 & 0 & 3 & 3 & 3 & 3 & 3 & 3 & 6 & 6 \\
\hline * & 6 & 5 & 4 & 3 & 3 & 3 & 3 & 3 & 3 & 6 & 6 & 6 \\
* & 6 & 5 & 4 & 3 & 3 & 3 & 3 & 3 & 6 & 6 & 6 & 6 \\
\hline * & * & * & * & * & 9 & 8 & 7 & 6 & 6 & 6 & 6 & 6 \\
* & * & * & * & * & 9 & 8 & 7 & 6 & 6 & 6 & 6 & 6
\end{array}\right) \quad\left(\begin{array}{lll|ll|ll}
0 & 0 & 0 & 0 & 0 & 0 & 2 \\
0 & 0 & 0 & 0 & 0 & 2 & 2 \\
0 & 0 & 0 & 0 & 2 & 2 & 2 \\
0 & 0 & 0 & 2 & 2 & 2 & 2 \\
\hline 4 & 3 & 2 & 2 & 2 & 2 & 2 \\
4 & 3 & 2 & 2 & 2 & 2 & 4 \\
4 & 3 & 2 & 2 & 2 & 4 & 4 \\
\hline * & * & 6 & 5 & 4 & 4 & 4 \\
* & * & 6 & 5 & 4 & 4 & 4 \\
* & * & 6 & 5 & 4 & 4 & 4
\end{array}\right)
$$

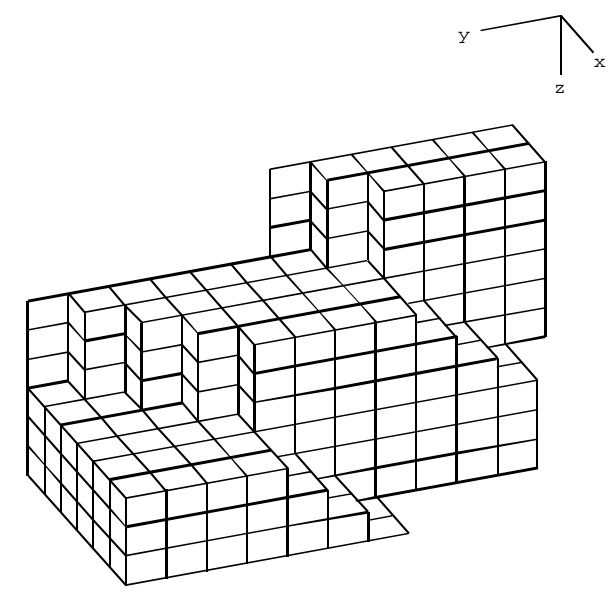

FiguRE 4. The 3-diagram for the $(2,4,3)$-tennis ball problem.

We now show that the matrix $\mathcal{M}_{n}$ gives the $n$-diagram and, moreover, that all paths contained in the $n$-diagram are $n$-configuration paths.

Theorem 3.4. The $n$-diagram for the $\left(l_{1}, l_{2}, l_{3}\right)$-tennis ball problem is given by the matrix $\mathcal{M}_{n}$. Furthermore, the $n$-configuration paths are exactly those contained in the $n$-diagram.

Proof. The proof is by induction on $n$. As seen above the case $n=1$ is trivial. Assume $\mathcal{M}_{n-1}$ is the matrix of $(n-1)$-diagram for the $\left(l_{1}, l_{2}, l_{3}\right)$-tennis ball problem. Let $\mathcal{N}$ be the matrix of the $n$-diagram; we prove that $\mathcal{N}=\mathcal{M}_{n}$. The matrix $\mathcal{N}$ has dimensions $\left(n l_{1}+1\right) \times\left(n l_{2}+1\right)$. 

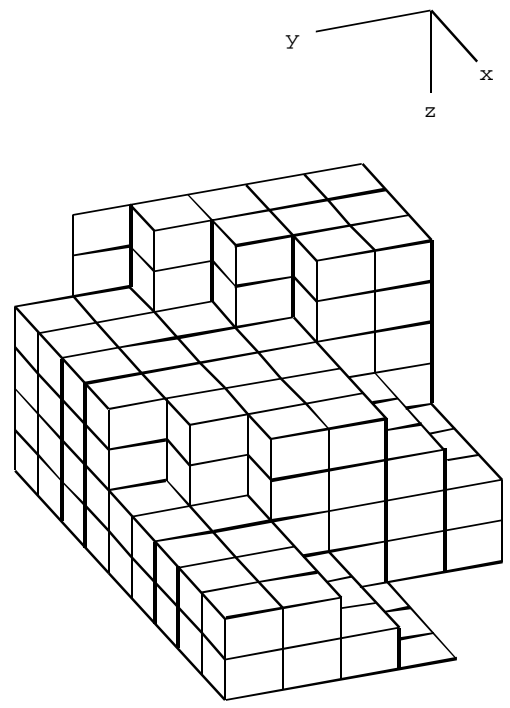

FiguRE 5. The 3-diagram for the (3,2,2)-tennis ball problem.

Recall that the entry in row $x+1$ and column $y+1$ of $\mathcal{N}$ is $m_{n}(x, y)$. Given $x$ and $y$ with $0 \leq x \leq n l_{1}$ and $0 \leq y \leq n l_{2}$, let $z$ be $m_{n}(x, y)$. We show that $z$ is the entry in row $x+1$ and column $y+1$ of the matrix $\mathcal{M}_{n}$. The proof has three cases.

Case 1. $x \leq(n-1) l_{1}$ and $y \leq(n-1) l_{2}$

In this case we need to show that $z=m_{n-1}(x, y)$. Let $\pi$ be an $(n-1)$ configuration path that contains the point $\left(x, y, m_{n-1}(x, y)\right)$. By Corollary 3.2 , $\pi$ can be extended to an $n$-configuration path, hence $z \leq m_{n-1}(x, y)$. To show equality, assume $z<m_{n-1}(x, y) \leq(n-1) l_{3}$ and let $\rho$ be an $n$-configuration path that contains the point $(x, y, z)$. Let $\rho^{\prime}$ be the initial segment corresponding to the first $x+y+z$ steps. By Corollary 3.2 again, $\rho^{\prime}$ can be extended to an $(n-1)$-configuration path contradicting the induction hypotheses.

Case 2. $x>(n-1) l_{1}$

We want to show in this case that $z$ is given by the entries of the matrices $C_{n}$ and $D_{n}$, depending on the value of $y$. Observe first that Lemma 3.1 implies that $x+y+z>(n-1) L$ and hence also that $y+z \geq(n-1)\left(l_{2}+l_{3}\right)$.

We discuss now the subcase $y \geq(n-1) l_{2}$, showing that $z=(n-1) l_{3}$. Let $\pi$ be any $(n-1)$-configuration path. Extend $\pi$ by adding $y-(n-1) l_{2}$ steps $e_{2}$ followed by $x-(n-1) l_{1}$ steps $e_{1}$ and then $l_{3}$ steps $e_{3}$, and add the remaining steps in any way. This path clearly satisfies the condition on Lemma 3.1 hence $z \leq(n-1) l_{3}$. To complete the proof of the claim, suppose that $z<(n-1) l_{3}$. Then by Lemma $3.1 x+y+z<(n-1) L$. This contradicts the first conclusion of the previous paragraph.

The other subcase left is $y<(n-1) l_{2}$. We show that in this case $z=(n-$ 1) $\left(l_{2}+l_{3}\right)-y$. Since we already know that $z$ is at least $(n-1)\left(l_{2}+l_{3}\right)-y$ it is enough to show that there is an $n$-configuration path containing the point 
$\left(x, y,(n-1)\left(l_{2}+l_{3}\right)-y\right)$. Consider the path

$$
\sigma=\left(e_{3}\right)^{(n-1)\left(l_{2}+l_{3}\right)-y}\left(e_{2}\right)^{y}\left(e_{1}\right)^{x}\left(e_{3}\right)^{n l_{3}-(n-1)\left(l_{2}+l_{3}\right)+y}\left(e_{2}\right)^{n l_{2}-y}\left(e_{1}\right)^{n l_{1}-x} .
$$

It is easy to check that this path satisfies the condition of Lemma 3.1 and hence it is an $n$-configuration path containing the point $\left(x, y,(n-1)\left(l_{2}+l_{3}\right)-y\right)$, as required.

Case 3. $x<(n-1) l_{1}$ and $y>(n-1) l_{2}$

In this case the value of $z$ has to be the one given by the matrix $B_{n}$. We have to show that if $s$ is such that $(n-s)\left(l_{1}+l_{2}\right) \leq x+y<(n-s+1)\left(l_{1}+l_{2}\right)$ then $z=(n-s) l_{3}$.

Since from Case 2 we have that the point $\left(n l_{1}, y,(n-1) l_{3}\right)$ is in the $n$-diagram, by Corollary 3.3 it follows that the point $\left(x, y,(n-1) l_{3}\right)$ is in the $n$-diagram as well, hence $z \leq(n-1) l_{3}$. If $x+y>(n-1)\left(l_{1}+l_{2}\right)$, then by Lemma 3.1 $x+y+z>(n-1) L$ and hence $z \geq(n-1) l_{3}$, so in this case $z=(n-1) l_{3}$.

Now suppose $(n-s)\left(l_{1}+l_{2}\right)<x+y \leq(n-s+1)\left(l_{1}+l_{2}\right)$. As in the previous paragraph, Lemma 3.1 implies that $z \geq(n-s) l_{3}$. To show that we have equality, we give an $n$-configuration path containing the point $\left(x, y,(n-s) l_{3}\right)$. Consider the path

$$
\sigma=\left(e_{3}\right)^{(n-s) l_{3}}\left(e_{2}\right)^{y}\left(e_{1}\right)^{x}\left(e_{3}\right)^{s l_{3}}\left(e_{2}\right)^{n l_{2}-y}\left(e_{1}\right)^{n l_{1}-x} .
$$

By using that $y>(n-1) l_{2}$ and that $x+y \leq(n-s+1)\left(l_{1}+l_{2}\right)$ it is easy to show that $\sigma$ satifies the condition of Lemma 3.1 and hence it is an $n$-configuration path.

To finish the proof we have to show that any path contained in the $n$-diagram is an $n$-configuration path. Let $\pi$ be such a path; we check that $\pi$ satisfies the condition in Lemma 3.1. Let $(X, Y, Z)$ be a point in the path with $X+Y+Z=t L$ for some $t$ with $1 \leq t \leq n-1$; our goal is to show that $X \leq t l_{1}$ and $X+Y \leq$ $t\left(l_{1}+l_{2}\right)$. Consider the point $p=\left(X, Y, m_{n}(X, Y)\right)$. The proof above shows that there is an $n$-configuration path $\pi^{\prime}$ that goes through $p$; since $Z \geq m_{n}(X, Y)$, we can apply Corollary 3.2 to obtain from $\pi^{\prime}$ an $n$-configuration path containing the point $(X, Y, Z)$. Since all $n$-configuration paths satisfy the condition in Lemma 3.1 , we have that $X \leq t l_{1}$ and $X+Y \leq t\left(l_{1}+l_{2}\right)$.

\section{Concluding Remarks}

The results of the previous section show that some sets of lattice paths in 3 dimensions can be interpreted in terms of flag matroids; hence, the flag matroids one obtains from the tennis ball problem naturally generalize lattice path matroids. This might lead to the suspicion that any set of paths in $\mathbb{N}^{k}$ with a "reasonable" border also gives rise to flag matroids. Unfortunately, it is very easy to produce counterexamples to this. For instance, consider the diagram in Figure 6. If the paths contained in that diagram were in correspondence with the flag bases of a flag matroid $F$, we would have that $B_{1}=\{2,6\}$ and $B_{2}=\{4,5\}$ are cobases of the second constituent of $F$. Hence it should be possible to replace 2 in $B_{1}$ by either 4 or 5 . But no path contained in the diagram has $\{4,6\}$ or $\{5,6\}$ as its set of steps in the direction $e_{3}$. 


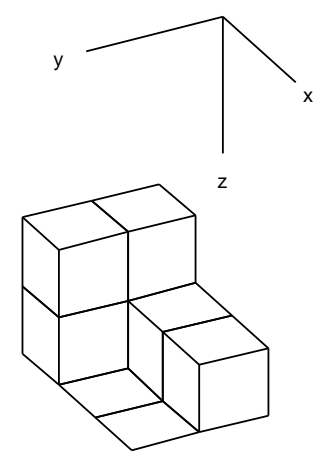

Figure 6 . The set of paths contained in the diagram does not give rise to a flag matroid.

A question that remains open is to solve the $\left(l_{1}, \ldots, l_{k}\right)$-tennis ball problem, or even the $(1,1,1)$-tennis ball problem. The approaches used previously to solve the case $k=2$ do not seem to generalize easily. In particular, the strategy from [7] would suggest the use of a Tutte polynomial-like invariant to count flag bases. There are some generalizations of the Tutte polynomial to pairs of matroids and to chains of matroids related by strong maps ([6, 11]), but unfortunately they do not seem to include the number of flag bases as an specialization. Following the Tutte polynomial approach for flag matroids would require defining first the suitable generalization.

We finish with some easy bounds. A trivial upper bound for the number of $n$-configurations is given by the total number of $\left(n l_{1}, \ldots, n l_{k}\right)$-partitions of $[n L]$, which is the multinomial coefficient

$$
\left(\begin{array}{c}
n L \\
n l_{1}, \ldots, n l_{k}
\end{array}\right)=\frac{(n L) !}{\left(n l_{1}\right) ! \cdots\left(n l_{k}\right) !} .
$$

The following connection with Young tableaux gives a lower bound on the number of $n$-configurations of the $\left(l_{1}, \ldots, l_{k}\right)$-tennis ball problem when $l_{k} \geq l_{k-1} \geq \cdots \geq l_{1}$. Consider sequences of length $n\left(l_{1}+\cdots l_{k}\right)$ over the alphabet $\left\{e_{1}, \ldots, e_{k}\right\}$ containing $n l_{i}$ copies of $e_{i}$ and such that in any initial subsequence the number of symbols $e_{i}$ is greater or equal than the number of symbols $e_{i-1}$, for all $i$ with $2 \leq i \leq$ $k$. Since all these sequences trivially satisfy the condition in Lemma 3.1, they give $n$-configuration paths. The number of such sequences equals the number of standard Young tableaux of shape $\left(n l_{k}, \ldots, n l_{1}\right)$, and this is given by the hooklength formula (see [9, Chapter 7]). In the case $l_{1}=\cdots=l_{k}=l$, this is

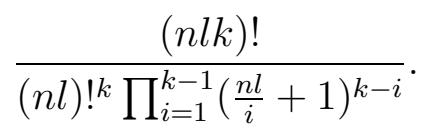

The general case gets more involved and we omit it since not much insight is gained.

For the $(1,1,1)$-tennis ball problem, the first order approximation of the lower and upper bounds are $C 27^{n} n^{-7 / 2}$ and $C^{\prime} 27^{n} n^{-1 / 2}$, respectively, for some constants 
$C$ and $C^{\prime}$. Computer evidence seems to suggest that the right number lies closer to the lower bound, and that the exponent in the term on $n$ is -3 ([10]).

A general lower bound for the $\left(l_{1}, \ldots, l_{k}\right)$-tennis ball problem can be obtained as follows. Let $t(a, b, n)$ be the number of $n$-configurations of the $(a, b)$-tennis ball problem. Then the number of $n$-configurations of the $\left(l_{1}, \ldots, l_{k}\right)$-tennis ball problem is at least

$$
t\left(l_{1}, l_{2}+\cdots+l_{k}, n\right) t\left(l_{2}, l_{3}+\cdots+l_{k}, n\right) \cdots t\left(l_{k-1}, l_{k}, n\right),
$$

since we can think of the $\left(l_{1}, \ldots, l_{k}\right)$-tennis ball problem as $n$ turns of the $\left(l_{1}, l_{2}+\right.$ $\left.\cdots+l_{k}\right)$-tennis ball problem, followed by $n$ turns of the $\left(l_{2}, l_{3}+\cdots+l_{k}\right)$-tennis ball problem on the result of the first, and so on. The bound is strict since each $t(a, b, n)$ counts the number of $n$-configurations of the $(a, b)$-tennis ball problem, but each of these can usually be reached by several movements of the balls and that is relevant for the version with $k$ bins.

\section{ACKNOWLEDGEMENTS}

The author thanks Dominic Welsh for asking a question that suggested this work, and Joseph Bonin and Sergi Elizalde for helpful discussions.

\section{REFERENCES}

[1] J. Bonin and A. de Mier, Lattice path matroids: structural properties, European J. Combin. 27 (2006), 701-738.

[2] J. Bonin, A. de Mier, and M. Noy, Lattice path matroids: enumerative aspects and Tutte polynomials, J. Combin. Theory Ser. A 104 (2003), 63-94.

[3] A. V. Borovik, I. M. Gelfand, A. Vince, and N. White, The lattice of flats and its underlying flag matroid polytope, Ann. Combin 1 (1997), 17-26.

[4] A. V. Borovik, I. M. Gelfand, and N. White, Coxeter matroids, Progress in Mathematics 216 (Birkhauser Boston, Inc., Boston, MA, 2003).

[5] V. Bryant and I. Sharpe, Gaussian, Strong and Transversal Greedoids, Europ. J. Combinatorics 20 (1999), 259-262.

[6] M. Las Vergnas, On the Tutte polynomial of a morphism of matroids, Ann. Discrete Math 8 (1980), 7-20.

[7] A. de Mier and M. Noy, A solution to the tennis ball problem, Theoret. Comput. Science 346 (2005), 254-264.

[8] J. G. Oxley, Matroid Theory, (Oxford University Press, Oxford, 1992).

[9] R. P. Stanley, Enumerative combinatorics, Vol. 2, Cambridge University Press, Cambridge, 1999.

[10] D. van der Zypen, private communication.

[11] D. Welsh and K. Kayibi, A linking polynomial of two matroids, Adv. in Appl. Math. 32 (2004), 391-419.

Mathematical Institute, University of Oxford, 24-29 St Giles, Oxford OX1 3LB, United Kingdom

E-mail address: ademier@gmail.com 